\title{
Dysregulation of microRNAs in triple-negative breast cancer
}

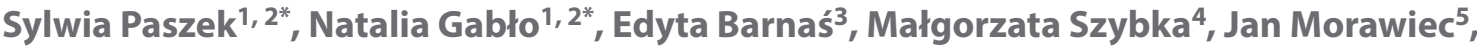 \\ Agnieszka Kołacińska ${ }^{5,6}$, Izabela Zawlik ${ }^{1,2}$ \\ ${ }^{1}$ Centre for Innovative Research in Medical and Natural Sciences, Faculty of Medicine, University of Rzeszow, Poland \\ ${ }^{2}$ Department of Genetics, Faculty of Medicine, University of Rzeszow, Poland \\ ${ }^{3}$ Institute of Obstetrics and Emergency Medicine, Faculty of Medicine, University of Rzeszow, Poland \\ ${ }^{4}$ Department of Microbiology and Laboratory Medical Immunology, Faculty of Medicine, Medical University of Lodz, Poland \\ ${ }^{5}$ Department of Head and Neck Cancer Surgery, Medical University of Lodz, Poland \\ ${ }^{6}$ Department of Surgical Oncology, Cancer Center, Copernicus Memorial Hospital, Poland \\ ${ }^{*}$ Equal contribution
}

\begin{abstract}
Objectives: Triple-negative breast cancer (TNBC) is an aggressive subtype of breast cancer with limited treatment options and poor prognosis. TNBC is usually diagnosed at a relatively young age and is characterized by high risk of developing metastases. Some epigenetic regulation of gene expression is associated with TNBC. Expression of microRNAs (miRNAs) can serve as a potential tool for identifying critical biomarkers in TNBC.

The aim of our study is to examine expression of selected miRNAs in TNBC and to assess the relationship between miRNA expression and clinicopathological factors.

Material and methods: Expression levels of 19 selected miRNAs were compared between cancerous and normal breast tissues by use of qPCR method. We have evaluated the relationship between the expression level of miRNAs and clinicopathological factors such as: age, tumor size and lymph node status.

Results: We found that in TNBC tissues, when compared with normal breast tissues, the expression of miR-190a, miR$136-5 p$ and miR-126-5p was significantly reduced $(p=0.0041, p=0.0007, p=0.0007$, respectively) whereas expression of miR-135b-5p and miR-182-5p was significantly increased ( $p=0.0194, p=0.0041$, respectively). We found a linear trend for tumor size and expression of miR-126-5p ( $p=0.0296)$ and miR-135b-5p ( $p=0.0241)$.

Conclusions: Our study confirms that miRNA expression profile is dysregulated in TNBC patients compared to healthy controls. MiR-190a, miR-136-5p, miR-126-5p, miR-135b-5p and miR-182-5p may be associated with development and progression of TNBC.
\end{abstract}

Key words: TNBC, microRNA expression, biomarkers

Ginekologia Polska 2017; 88, 10: 530-536

\section{INTRODUCTION}

Breast cancer is a heterogeneous disease and a major cause of female mortality [1]. Triple-negative breast cancer (TNBC) is defined by a lack of expression of estrogen receptor (ER), progesterone receptor (PR) and of human epidermal growth factor receptor-2 (HER2) [2]. TNBC accounts for approximately $15-25 \%$ of all breast cancer types and is an aggressive histological subtype. There exist following
TNBC subtypes: basal or BRCA-related, apocrine, normal-like and other rare subtypes including a neuroendocrine TNBC [3]. Subtyping of TNBC is necessary to choose an optimal molecular-based therapy [4]. TNBC is mostly diagnosed in patients at a relatively young age and is characterized by frequent metastasis [5, 6]. Patients with TNBC have increased risk of relapse and tend to resist to chemotherapies [7]. Thus, further subtyping with more individual therapy adjustments 
could improve patients'survival. One of the new potentially useful biomarkers in oncology is miRNA [8].

miRNAs are short non-coding RNAs of 19-25 nucleotides in length. They regulate gene expression post-transcriptionally by pairing with complementary nucleotide sequences in the 3'-UTRs of specific mRNA targets [9]. Deregulation of specific miRNAs associated with carcinogenesis causes changes in their expression and function. This way some acquire the features of oncogenes while others become tumor suppressors [10]. Since 1993, when the first miRNA lin- 4 was discovered, the knowledge about miRNAs has been greatly expanded. The regulatory role of miRNA was determined in many cellular pathways involved in cell division, differentiation, morphogenesis, metabolism and apoptosis [11]. Studies carried out on various types of cancers made clear that miRNA expression is an important factor for the carcinogenesis. TNBC is a heterogeneous subtype of breast cancer that has limited treatment options [12]. Thus, miRNA studies may be useful for developing new therapeutic strategies. It has been reported that numerous cellular signaling pathway abnormalities occur in TNBC [13]. There has been increasingly more evidence supporting the role of miRNAs in cell signaling pathways of triple-negative breast cancer but its clinical significance is still unclear $[14,15]$.

\section{OBJECTIVES}

The aim of our study is to examine the expression of selected miRNAs in TNBC tissues in comparison with normal breast tissue and to assess the relationship between miRNA expression and some clinical features such as: age of onset, tumor size and lymph node status.

\section{MATERIAL AND METHODS}

The study was conducted under Institutional Review Board protocol \#RNN/226/11/KE/13/12/2011 at Medical University of Lodz. All patients gave written informed consent. We included in this study 11 TNBC female patients and 3 healthy age-matched women controls. All of 11 examined patients exhibit an invasive ductal histology and a third histologic grade. One of the patients had evidence of distant metastases in lungs and liver. Between December 2011 and April 2012 specimens were collected at the Cancer Center with ultrasound-guided 14-gauge core needle biopsy (ultra-automatic biopsy instrument Pro-Mag TM, Angiotech) from 11 female patients with either inoperable locally advanced breast cancer or with large operable tumor suitable for down-staging. Further specimens were obtained from 3 healthy age-matched women controls (breast reduction procedures). Four to five specimens were obtained from each lesion. Half of samples were frozen immediately at $-80^{\circ} \mathrm{C}$ while the rest was paraffin embedded and reviewed by breast pathology specialist in the Department of Pathol- ogy. Estrogen receptor (ER) and progesterone receptor (PR) status were determined by immunohistochemistry (IHC) using the Allred score. Human epidermal growth factor receptor 2 (HER2) status was evaluated by immunohistochemistry or by fluorescence in situ hybridization. Samples were considered ER or PR negative if less than $1 \%$ of the tumor cells were immunoreactive. Samples were considered HER2 negative with IHC 1+ staining or with a score of 2+ and no HER2 gene amplification when tested by FISH. TNBC was diagnosed when result was ER, PR and HER2 negative.

\section{MicroRNA expression analysis by use of qPCR}

Selection of 19 miRNAs was performed on the basis of previous reports in the literature and the miRNA database. In the total of 14 breast tissue samples (11 from TNBC and 3 from healthy controls) we analyzed $19 \mathrm{mi}-$ RNAs: hsa-miR-512-5p, hsa-miR-190a, hsa-miR-200b-3p, hsa-miR-622, hsa-miR-346, hsa-miR-148b-5p, hsa-miR-449a, hsa-miR-1255b-5p, hsa-miR-203a, hsa-miR-577, hsa-miR93-5p, hsa-miR-126-5p, hsa-miR-423-5p, hsa-miR-129-5p, hsa-miR-193b-5p, hsa-miR-182-5p, hsa-miR-136-5p, hsamiR-191-5p and hsa-miR-135b-5p. Hsa-miR-103a-3p and hsa-miR-107 were chosen for normalization, which was further validated by NormFinder algorithm. RNA was isolated using miReasy Mini Kit 50 (Qiagen, Valencia, CA, USA). $10 \mathrm{ng}$ RNA sample was reverse transcribed in $10 \mu \mathrm{l}$ reactions using the miRCURY LNA ${ }^{\text {TM }}$ Universal RT microRNA PCR, Polyadenylation and CDNA synthesis kit (Exiqon, Copenhagen, Denmark). Each RT was performed in duplicates. cDNA was diluted $100 \times$ and assayed in $10 \mu \mathrm{L}$ PCR reactions according to the protocol for miRCURY LNA ${ }^{\mathrm{TM}}$ Universal RT microRNA PCR; each miRNA was assayed once by qPCR on the microRNA Ready-to-Use PCR, Custom Pick\&Mix panel. Negative controls excluding templates from the reverse transcription reaction were processed and profiled in a similar fashion. Amplification was performed in a LightCycler ${ }^{\circledR} 480$ Real-Time PCR System (Roche, Basel, Switzerland). The amplification curves were analyzed using the Roche LC software, both for determination of $\mathrm{Cp}$ (by the 2 nd derivative method) and for melting curve analysis.

\section{Data analysis}

The assay efficiency was determined by means of analysis of the amplification curves using algorithms similar to the LinReg software. Individual reactions that gave efficiency of $<1.6$ were excluded from the dataset. The efficiencies ranged between 1.8 and 2.1. All assays were inspected for distinct melting curves and the Tm was confirmed to be within known specifications for the assay. It was important that assays with $5 \mathrm{Cp}$ 's less compared to negative control be detected and those with $\mathrm{Cp}<37$ be included in the data analysis. Data that failed to reach such criteria was 
excluded from further analysis. The stability of miRNAs was measured by NormFinder software [16]. For normalization of the data we have used the average of hsa-miR-103a-3p and hsa-miR-107, which proved to be the most stable normalizer (according to E normalization). (Stability value for best combination of such pair equals 0.045). The formula used to calculate the normalized $\mathrm{Cp}$ values was:

Normalized delta $C p=$ average $C p$ (hsa-miR-103a-3p and hsa-miR-107) — assay Cp (sample).

\section{Target gene selection}

In order to find miRNA target genes we have searched miRTarBase 6.0 - a database that manually collects experimental data about miRs-genes interactions. We selected only those interactions concerning human miRNAs and human gene targets proved by strong experimental evidence method (Reporter assay, Western blot or qPCR technique) [17].

\section{Statistical analysis}

Continuous variables were presented as mean values with respective standard deviations. Selected miRNA expression levels in TNBC tissues and normal tissues were compared using a Student t-test after adjustment for Benjamini-Hochberg multiple comparison (False Discovery Rate). Assessment of correlation between a particular miRNA expression and the patients' age was performed with Pearson correlation. Association between tumor size (T stage) and lymph node status ( $\mathrm{N}$ stage) was verified by linear trend analysis. For multidimensional data - Principal Component Analysis and Unsupervised Hierarchical Clustering was performed. Statistical analysis was done with MultiExperiment Viewer (MeV) software (Dana Farber, Boston, USA) and STATISTICA 10.0 software (Statsoft, Tulsa, OK, USA). P values lower than 0.05 were considered as statistically significant.

\section{RESULTS}

Ten common miRNAs were identified in all samples with an average of 18 miRNAs detectable per sample. MicroRNAs detected in all control samples and in more than 8 TNBC samples were further analyzed. Thus, 14 miRNAs have been included in the Principal Component Analysis (PCA) (Fig. 1) and in the two-way hierarchical clustering of miRNAs (Fig. 2). Samples seem to cluster based on group information provided; however, sample 9 falls closer to the normal tissue samples than to the TNBC (Fig. 1 and Fig. 2).

The analysis revealed that five miRNAs (miR-136-5p, miR-126-5p, miR-182-5p, miR-190a, miR-135b-5p) have a significantly different expression in TNBC tissue when compared with healthy controls (Table 1). We observed a significant down-regulation of miR-190a, miR-136-5p and miR-126-5p (dCp $-5.95 \pm 0.91$ vs. $-4.36 \pm 0.28$,

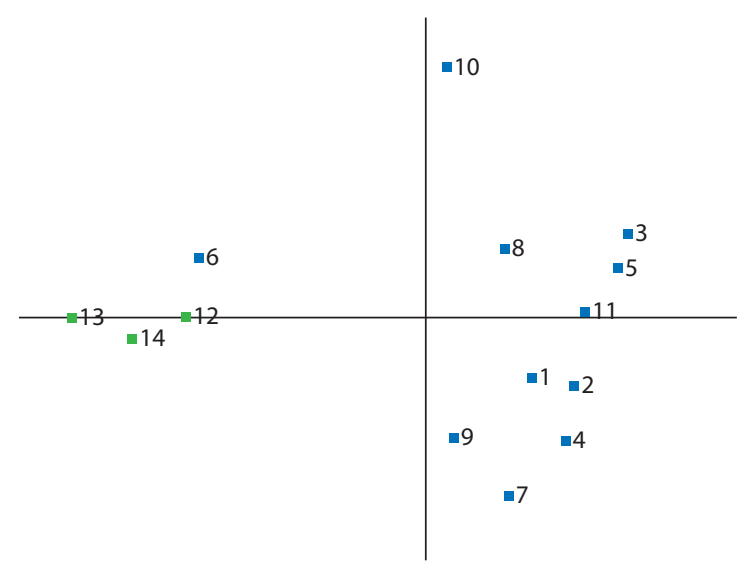

Figure 1. The Principal Component Analysis plot. PCA was performed on all samples and on those assays with a count of at least 12 (missing values are substituted by group averages). The normalized $(\mathrm{dCp})$ values have been used for the analysis. The analysis was performed on 3 normal breast tissues (green color) and 11 TNBC tissues (blue color)

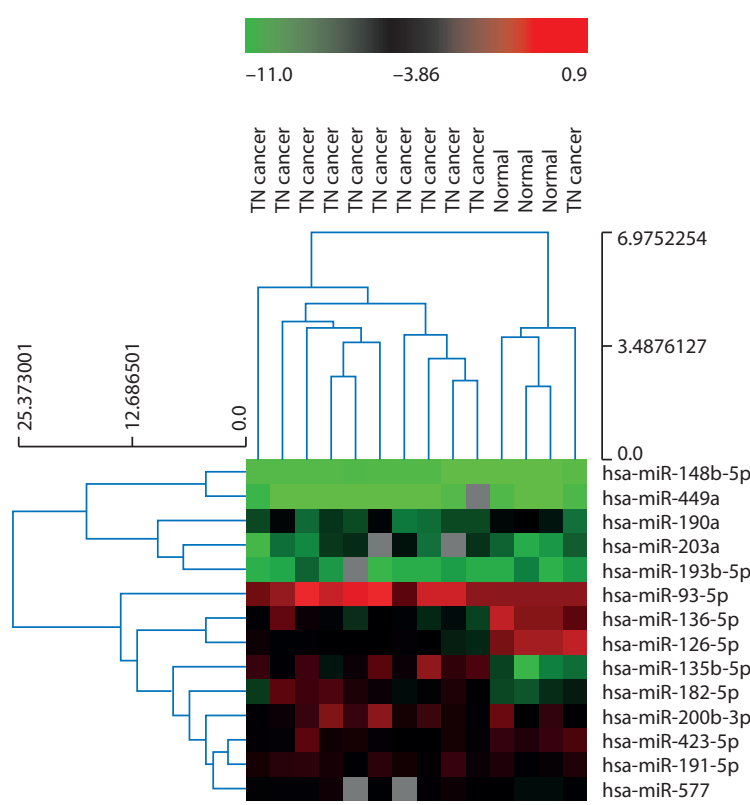

Figure 2. Heat map and unsupervised hierarchical clustering. The clustering is performed on all samples and on those assays with a count of at least 12 . The color scale illustrates the relative expression level of microRNA across all samples: red color represents an expression level above mean, green color represents expression lower than the mean. The normalized $(\mathrm{dCp})$ values have been used for the analysis

$p=0.0041, d C p-4.03 \pm 1.21$ vs. $-0.94 \pm 0.39, p=0.0007$, $\mathrm{dCp}-3.61 \pm 1.18$ vs. $-1.06 \pm 0.31, p=0.0007$, respectively). The following in turn were up-regulated: miR-135b-5p and miR-182-5p (dCp -3.13 \pm 1.44 vs. $-7.08 \pm 0.91, p=0.0194$, $d C p-3.48 \pm 1.13$ vs. $-5.79 \pm 0.41, p=0.0041$, respectively) (Table 1). The association between tumor size ( $T$ ), lymph node status $(\mathrm{N})$ and patient's age and miRNAs expression is presented in Table 2 . 
Table 1. Selected miRNAs expressed in normal breast tissues and in TNBC tissues

\begin{tabular}{|c|c|c|c|c|c|}
\hline \multirow{2}{*}{ miRNA names } & \multirow{2}{*}{ Count } & \multicolumn{2}{|c|}{ Average $\mathrm{dCp} \pm$ standard deviation } & \multirow{2}{*}{ p-value } & \multirow{2}{*}{$\mathrm{FDR}^{*}$} \\
\hline & & TNBC patients & Healthy controls & & \\
\hline hsa-miR-136-5p & 14 & $-4.03 \pm 1.21$ & $-0.94 \pm 0.39$ & 7.34E-05 & 0.0007 \\
\hline hsa-miR-126-5p & 14 & $-3.61 \pm 1.18$ & $-1.06 \pm 0.31$ & $8.89 \mathrm{E}-05$ & 0.0007 \\
\hline hsa-miR-182-5p & 14 & $-3.48 \pm 1.13$ & $-5.79 \pm 0.41$ & 0.0008 & 0.0041 \\
\hline hsa-miR-190a & 14 & $-5.95 \pm 0.91$ & $-4.36 \pm 0.28$ & 0.0010 & 0.0041 \\
\hline hsa-miR-135b-5p & 14 & $-3.13 \pm 1.44$ & $-7.08 \pm 0.91$ & 0.0061 & 0.0194 \\
\hline hsa-miR-577 & 12 & $-3.57 \pm 0.34$ & $-4.37 \pm 0.29$ & 0.0333 & 0.0888 \\
\hline hsa-miR-423-5p & 14 & $-3.17 \pm 0.54$ & $-2.70 \pm 0.15$ & 0.0415 & 0.0948 \\
\hline hsa-miR-93-5p & 14 & $-0.40 \pm 1.04$ & $-1.14 \pm 0.05$ & 0.0488 & 0.0977 \\
\hline hsa-miR-203a & 12 & $-6.44 \pm 1.38$ & $-7.65 \pm 0.78$ & 0.1547 & 0.2751 \\
\hline hsa-miR-148b-5p & 14 & $-10.65 \pm 0.48$ & $-11.09 \pm 0.33$ & 0.1878 & 0.3005 \\
\hline hsa-miR-191-5p & 14 & $-3.00 \pm 0.28$ & $-3.23 \pm 0.23$ & 0.2984 & 0.3979 \\
\hline hsa-miR-449a & 13 & $-11.03 \pm 0.75$ & $-11.21 \pm 0.82$ & 0.7935 & 0.8521 \\
\hline hsa-miR-200b-3p & 14 & $-2.70 \pm 0.76$ & $-2.88 \pm 0.85$ & 0.7989 & 0.8521 \\
\hline hsa-miR-193b-5p & 13 & $-8.09 \pm 0.59$ & $-8.08 \pm 0.58$ & 0.9832 & 0.9832 \\
\hline
\end{tabular}

*False Discovery Rate

Table 2. P values for association of miRNA expression with patient age, tumor size (T stage) and lymph node status ( $\mathrm{N}$ stage)

\begin{tabular}{|l|c|c|c|}
\hline miRNA name & $\mathbf{T}$ & $\mathbf{N}$ & Age \\
\hline hsa-miR-136-5p & 0.0681 & 0.4447 & 0.4627 \\
\hline hsa-miR-126-5p & 0.0296 & 0.7999 & 0.4627 \\
\hline hsa-miR-182-5p & 0.1491 & 0.1186 & 0.7473 \\
\hline hsa-miR-190a & 0.5595 & 0.8718 & 0.6667 \\
\hline hsa-miR-135b-5p & 0.0241 & 0.5629 & 0.7678 \\
\hline
\end{tabular}

[For T and N, p value was calculated with linear trend whereas for Age with Spearman correlation]
A

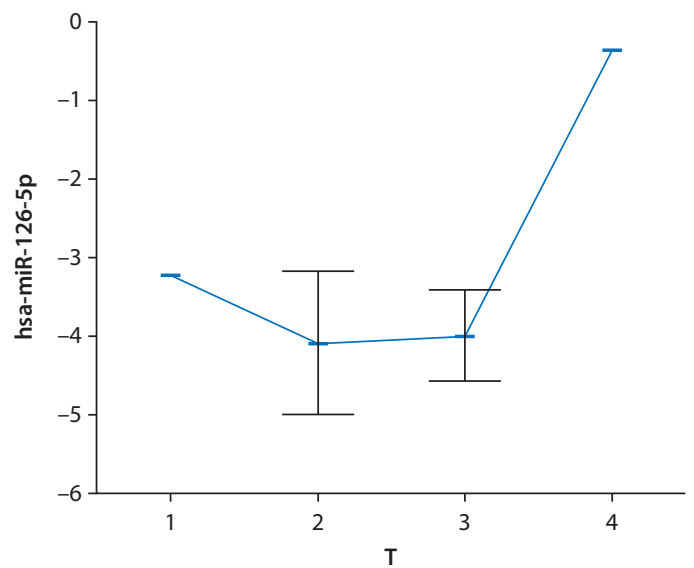

We noticed a significant linear trend for $T$ value and expression levels of miR-126-5p ( $p=0.0296)$ and miR-135b-5p $(p=0.0241)$ (Figure 3). Moreover, miR-126-5p correlated positively with expression of miR-136-5p $(r=0.61, p=0.0444)$ and negatively with miR-135b-5p $(r=(-0.72), p=0.0124)$.

Moreover, we searched for 5 selected miRNA validated target genes. MiRTarBase has showed associations with 50 genes with strong evidenced for being targets for one of the 5 miRNAs (Table 3).

B

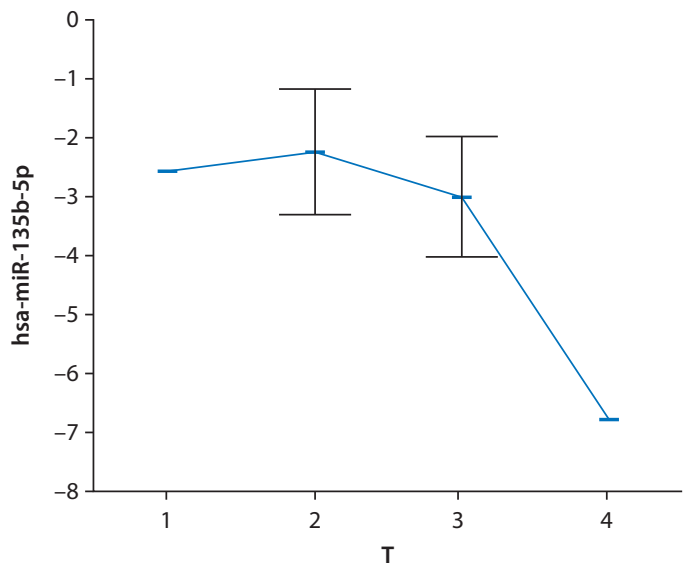

Figure 3. Association between hsa-miR-126-5p (A), hsa-miR-135b-5p (B) and tumor size (T stage) 
Table 3. Human target genes for significantly dysregulated human microRNAs in a TNBC from miRTarBase, confirmed by strong evidence validation methods (Reporter assay, Western blot, qPCR)

\begin{tabular}{l|l}
\hline MicroRNA name & Target gene name \\
\hline hsa-miR-136-5p & MTDH, BCL2, PPP2R2A \\
\hline hsa-miR-126-5p & SLC45A3, PTPN7, ADAM9, MMP7, CXCL12, MYC \\
\hline hsa-miR-182-5p & $\begin{array}{l}\text { CDKN1A, FOXO3, MTSS1, FOXO1, RARG, MITF, ADCY6, CLOCK, TSC22D3, CREB1, FGF9, NTM, CYLD, BCL2, CCND2, PDCD4, } \\
\text { PFN1, SNAI2, RECK, SMAD4, FOXF2, FLOT1, PTEN, GSK3B, ANUBL1, SATB2, CHL1, CADM1, TP53INP1, ATF1, BARD1, CREB5, } \\
\text { RAD17, TP53BP1, CHEK2, CDKN1B, SMARCD3, TCEAL7, FBXW7, LRRC4, NDRG1, THBS1, ULBP2, BDNF, EP300 }\end{array}$ \\
\hline hsa-miR-190a-5p & CDKN1B, PHLPP1, KCNQ5, MARK2, IGF1 \\
\hline hsa-miR-135b-5p & APC, KLF4, MAFB, CASR, PPP2R5C, SMAD5, LZTS1, MID1, MTCH2, ACVR1B, BMPR2, TGFBR1, IBSP, BGLAP, RUNX2, SP7
\end{tabular}

\section{DISCUSSION}

Wefound that expression of miR-182-5pand miR-135b-5p was significantly increased while that of miR-190a, miR-136-5p and miR-126-5p was significantly reduced in TNBC tissues in comparison with normal breast tissues. We observed a linear trend for $T$ stage and expression of miR-126-5p and miR-135b-5p. In the study we did not find a significant association between the expression level of miRNAs and patients' age of onset or lymph node status.

MiR-182-5p belongs to themiR-183 family and miR-182-5p is encoded on chromosome 7q31-34 [18]. Medimegh et al. (2014) examined expression of miR-182 in the following three groups: TNBC, NTNBC (Non Triple Negative Breast Cancer; Luminal A, Luminal B and HER2 positive patients) and healthy controls. The analysis showed significantly higher expression of miR-182 in TNBC with respect to NTNBC cases. Moreover, the expression of miR-182 in both cancer groups was still higher than in healthy controls [19]. In present study we confirm the increased expression of miR-182-5p in TNBC patients.

It was reported that miR-182-5p is important for the tumor development as miR-182 has been associated with DNA damage repair, cell proliferation and apoptosis [20]. MiR-182 inhibits expression of $B R C A 1$ [21] that is responsible for repair of double-stranded DNA damage (DSBs) in homologous recombination (HR). Deficiency of BRCA1 protein leads to activation of other than HR repair mechanisms, the inefficiency of which causes genetics instability [22]. Moreover, silencing of miR-182-5p resulted in increase of BRCA1 levels and induction of resistance to radiation and to treatment with poly (ADP-ribose) polymerase 1 (PARP-1) inhibitors [21]. This phenomenon could be explained by activation of BRCA1-related tumor cell repair mechanism. Therefore, tumor cells do not activate the apoptotic pathway and become more resistant to treatment [23]. Medimegh et al. have noticed a significant association of lymph node metastases with miR-182 overexpression in TNBC of Tunisian population [19]. In present study we did not find any significant association between miR-182-5p expression and lymph node status, which is consistent with results of Krishnan et al. (2013) [24].
In our study we also noticed a significantly higher expression of miR-135-5p. The knowledge about miR-135$5 p$ function in TNBC development is limited. Up-regulated miR-135b was so far identified only in basal like subtype of breast cancer and estrogen-negative patients. In turn, miR-135b expression was correlated with worse survival of breast cancer patients and metastasis status [25]. In the study of Muñoz-Rodríguez et al. (2015) it was showed that miR-135b expression levels are higher in patients with breast cancer in early postpartum period compared to late postpartum period. Moreover, the miR-135b gene expression and its DNA methylation had a significant inverse correlation [26]. MiR-135b has been showed to be up-regulated in breast cancer [27], which is consistent with our study. Moreover miR-135b promotes cellular proliferation and disrupts the cell cycle of breast cancer cells by regulating LATS2 [27]. In our study we show a linear trend for T stage and expression of miR-135b-5p; we observe lower expression in higher tumor size.

Our study also showed that miR-190a, miR-126-5p and miR-136-5p are down-regulated in TNBC tissues. Hao et al. (2014) brought attention to the role of miR-190a in suppression of tumor metastasis via interaction with VEGF-mediated tumor angiogenesis [28]. In breast cancer, overexpression of miR-190a has inhibited the cell migration and invasiveness. Moreover, miR-190a expression in primary breast carcinomas correlated with overall survival [29]. Nevertheless, the role of miR-190a in breast cancer biology is still unclear.

We also report down-regulated miR-126-5p expression in TNBC. A different study has revealed that miR-126 plays a tumor-suppressor role and impedes the metastasis of non-small cell lung cancer by inhibiting tumor growth and cell invasion [30]. Moreover, miR-126 target genes include a variety of oncogenic factors such as ADAM9 [31]. The loss of miR-126 expression was associated with poor distal metastasis-free survival of breast cancer patients [32]. In our study there is linear trend for miR-126; we observed higher miR-126 levels in bigger tumor size.

When it comes to miR-136, it has been shown that it is up-regulated in lung cancer [33]. In turn, Zhao et al. ob- 
served low expression of miR-136 in patients with chemoresistant epithelial ovarian cancer [34]. Generally, it has been demonstrated that miR-136 was down-regulated in TNBC [35], which is consistent with our study. Yan et al. showed that suppression of tumor invasion and metastasis by miR-136 was mediated through targeting RASAL2, which plays oncogenic role in TNBC. Moreover, expression of miR136 was negatively correlated with WHO grades in TNBC [35].

In summary, the present study confirms the aberrant expression of miRNAs in TNBC patients when compared to healthy tissues. Such results indicate the influence of epigenetic factors in the development of TNBC. We have demonstrated a dysregulated expression of 5 miRNAs in TNBC tissues. Moreover, we have found a linear trend for tumor size and expression levels of miR-126-5p and miR-135b-5p.

Yet, it is necessary to verify our results on a larger population. The present study provides further experimental data for these candidate miRNAs in order to aid the identification of reliable molecular diagnostic markers of TNBC in the future.

\section{Acknowledgments}

This study was supported by the grant no. 2011/01/ /B/NZ4/03345 of the National Science Centre, Poland,. The study was performed within the project 'Centre for Innovative Research in Medical and Natural Sciences' realized by University of Rzeszow, co-financed within Regional Operational Program for the Podkarpackie Province for the years 2007-2013, contract number UDA-RPPK.01.03.00-18-004/12-00. The study was partly supported by funds from the University of Rzeszow, Faculty of Medicine. We would like to thank PhD Beata Małachowska for assistance with statistical analysis.

\section{Author disclosure statement}

None.

\section{REFERENCES}

1. Siegel R, Ma J, Zou Z, et al. Cancer statistics, 2014. CA Cancer J Clin. 2014; 64(1): 9-29, doi: 10.3322/caac.21208, indexed in Pubmed: 24399786.

2. Cheang MCU, Voduc D, Bajdik C, et al. Basal-like breast cancer defined by five biomarkers has superior prognostic value than triple-negative phenotype. Clin Cancer Res. 2008; 14(5): 1368-1376, doi: 10.1158/10780432.CCR-07-1658, indexed in Pubmed: 18316557.

3. Hudis CA, Gianni L. Triple-negative breast cancer: an unmet medical need. Oncologist. 2011; 16 Suppl 1: 1-11, doi: 10.1634/theoncologist.2011-S1-01, indexed in Pubmed: 21278435.

4. Godfrey SS, Moses MM, Rorie CJ. Cancer Associated MicroRNAs are Differentially Expressed in Triple Negative Breast Cancer and Normal Breast Cells. Int J Surg Res. 2015: 4-78.

5. Boyle P. Triple-negative breast cancer: epidemiological considerations and recommendations. Ann Oncol. 2012; 23 Suppl 6: vi7-v12, doi: 10.1093/annonc/mds187, indexed in Pubmed: 23012306.

6. Anders C, Carey LA. Understanding and treating triple-negative breast cancer. Oncology (Williston Park). 2008; 22(11): 1233-9; discussion 1239, indexed in Pubmed: 18980022.
7. O'Reilly EA, Gubbins $L$, Sharma $S$, et al. The fate of chemoresistance in triple negative breast cancer (TNBC). BBA Clin. 2015; 3: 257-275, doi: 10.1016/j.bbacli.2015.03.003, indexed in Pubmed: 26676166.

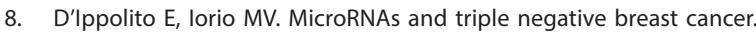
Int J Mol Sci. 2013; 14(11): 22202-22220, doi: 10.3390/ijms141122202, indexed in Pubmed: 24284394.

9. Pillai RS. MicroRNA function: multiple mechanisms for a tiny RNA? RNA. 2005; 11(12): 1753-1761, doi: 10.1261/rna.2248605, indexed in Pubmed: 16314451.

10. Dvinge $H$, Git $A$, Gräf $S$, et al. The shaping and functional consequences of the microRNA landscape in breast cancer. Nature. 2013; 497(7449): 378-382, doi: 10.1038/nature12108, indexed in Pubmed: 23644459.

11. HaTY. MicroRNAs in Human Diseases: From Cancer to Cardiovascular Disease. Immune Netw. 2011; 11(3): 135-154, doi: 10.4110/in.2011.11.3.135, indexed in Pubmed: 21860607.

12. Irshad S, Ellis P, Tutt A. Molecular heterogeneity of triple-negative breast cancer and its clinical implications. Curr Opin Oncol. 2011;23(6):566-577, doi: 10.1097/CCO.0b013e32834bf8ae, indexed in Pubmed: 21986848.

13. Hsu YH, Yao J, Chan LC, et al. Definition of PKC-a, CDK6, and MET as therapeutic targets in triple-negative breast cancer. Cancer Res. 2014; 74(17): 4822-4835, doi: 10.1158/0008-5472.CAN-14-0584, indexed in Pubmed: 24970481.

14. Avery-Kiejda KA, Braye SG, Mathe A, et al. Decreased expression of key tumour suppressor microRNAs is associated with lymph node metastases in triple negative breast cancer. BMC Cancer. 2014; 14: 51, doi: 10.1186/1471-2407-14-51, indexed in Pubmed: 24479446.

15. Matamala N, Vargas MT, González-Cámpora R, et al. MicroRNA deregulation in triple negative breast cancer reveals a role of miR-498 in regulating BRCA1 expression. Oncotarget. 2016; 7(15): 20068-20079, doi: 10.18632/oncotarget.7705, indexed in Pubmed: 26933805.

16. Andersen $C L$, Jensen JL, Ørntoft TF. Normalization of real-time quantitative reverse transcription-PCR data: a model-based variance estimation approach to identify genes suited for normalization, applied to bladder and colon cancer data sets. Cancer Res. 2004; 64(15): 5245-5250, doi: 10.1158/0008-5472.CAN-04-0496, indexed in Pubmed: 15289330.

17. Chou CH, Chang NW, Shrestha S, et al. miRTarBase 2016: updates to the experimentally validated miRNA-target interactions database. Nucleic Acids Res. 2016; 44(D1): D239-D247, doi: 10.1093/nar/gkv1258, indexed in Pubmed: 26590260.

18. Wang $\mathrm{F}$, Zhong S, Zhang H, et al. Prognostic Value of MicroRNA-182 in Cancers: A Meta-Analysis. Dis Markers. 2015; 2015: 482146, doi: 10.1155/2015/482146, indexed in Pubmed: 26063957.

19. Medimegh I, Omrane I, Privat $M$, et al. MicroRNAs expression in triple negative vs non triple negative breast cancer in Tunisia: interaction with clinical outcome. PLoS One. 2014; 9(11): e111877, doi: 10.1371/journal. pone.0111877, indexed in Pubmed: 25369070.

20. Wei $Q$, Lei $R, H u G$. Roles of miR-182 in sensory organ development and cancer. Thorac Cancer. 2015; 6(1): 2-9, doi: 10.1111/1759-7714.12164, indexed in Pubmed: 26273328.

21. Moskwa P, Buffa FM, Pan Y, et al. miR-182-mediated downregulation of BRCA1 impacts DNA repair and sensitivity to PARP inhibitors. Mol Cell. 2011;41(2): 210-220, doi: 10.1016/j.molcel.2010.12.005, indexed in Pubmed: 21195000.

22. Severson TM, Peeters J, Majewski I, et al. BRCA1-like signature in triple negative breast cancer: Molecular and clinical characterization reveals subgroups with therapeutic potential. Mol Oncol. 2015; 9(8): 1528-1538, doi: 10.1016/j.molonc.2015.04.011, indexed in Pubmed: 26004083.

23. de RuijterTC, Veeck J, de Hoon JPJ, et al. Characteristics of triple-negative breast cancer. J Cancer Res Clin Oncol. 2011; 137(2): 183-192, doi: 10.1007/s00432-010-0957-x, indexed in Pubmed: 21069385.

24. Krishnan K, Steptoe AL, Martin HC, et al. MicroRNA-182-5p targets a network of genes involved in DNA repair. RNA. 2013; 19(2): 230-242, doi: 10.1261/rna.034926.112, indexed in Pubmed: 23249749.

25. Arigoni M, Barutello G, Riccardo F, et al. miR-135b coordinates progression of ErbB2-driven mammary carcinomas through suppression of MID1 and MTCH2. Am J Pathol. 2013; 182(6): 2058-2070, doi: 10.1016/j. ajpath.2013.02.046, indexed in Pubmed: 23623609.

26. Muñoz-Rodríguez JL, Vrba L, Futscher BW, et al. Differentially expressed microRNAs in postpartum breast cancer in Hispanic women. PLoS One. 2015; 10(4): e0124340, doi: 10.1371/journal.pone.0124340, indexed in Pubmed: 25875827

27. Hua K, Jin J, Zhao J, et al. miR-135b, upregulated in breast cancer, promotes cell growth and disrupts the cell cycle by regulating LATS2. Int J Oncol. 2016; 48(5): 1997-2006, doi: 10.3892/ijo.2016.3405, indexed in Pubmed: 26934863 
28. Hao Y, Yang J, Yin S, et al. The synergistic regulation of VEGF-mediated angiogenesis through miR-190 and target genes. RNA. 2014; 20(8): 1328-1336, doi: 10.1261/rna.044651.114, indexed in Pubmed: 24962367.

29. Chu HW, Cheng CW, Chou WC, et al. A novel estrogen receptor-microRNA 190a-PAR-1-pathway regulates breast cancer progression, a finding initially suggested by genome-wide analysis of loci associated with lymph-node metastasis. Hum Mol Genet. 2014; 23(2): 355-367, doi: 10.1093/hmg/ddt426, indexed in Pubmed: 24009311.

30. Yang J, Lan $\mathrm{H}$, Huang $X$, et al. MicroRNA-126 inhibits tumor cell growth and its expression level correlates with poor survival in non-small cell lung cancer patients. PLoS One. 2012; 7(8): e42978, doi: 10.1371/journal. pone.0042978, indexed in Pubmed: 22900072.

31. Wang CZ, Yuan P, Li Y. MiR-126 regulated breast cancer cell invasion by targeting ADAM9. Int J Clin Exp Pathol. 2015; 8(6): 6547-6553, indexed in Pubmed: 26261534.
32. Tavazoie SF, Alarcón C, Oskarsson T, et al. Endogenous human microRNAs that suppress breast cancer metastasis. Nature. 2008; 451(7175): 147-152, doi: 10.1038/nature06487, indexed in Pubmed: 18185580.

33. Liu Xi, Sempere LF, Ouyang H, et al. MicroRNA-31 functions as an oncogenic microRNA in mouse and human lung cancer cells by repressing specific tumor suppressors. J Clin Invest. 2010; 120(4): 1298-1309, doi: 10.1172/JCl39566, indexed in Pubmed: 20237410.

34. Zhao H, Liu S, Wang G, et al. Expression of miR-136 is associated with the primary cisplatin resistance of human epithelial ovarian cancer. Oncol Rep. 2015; 33(2): 591-598, doi: 10.3892/or.2014.3640, indexed in Pubmed: 25482209.

35. Yan M, Li X, Tong D, et al. miR-136 suppresses tumor invasion and metastasis by targeting RASAL2 in triple-negative breast cancer. Oncol Rep. 2016; 36(1):65-71, doi: 10.3892/or.2016.4767, indexed in Pubmed: 27108696. 